\title{
Correction to: Responses of Microbial Community to Di-(2-ethylhcxyl) Phthalate Contamination in Brown Soil
}

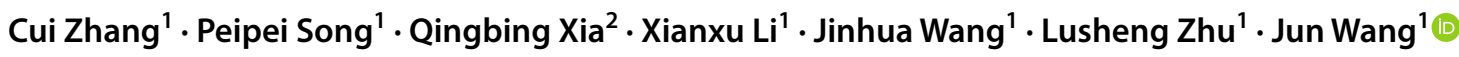

Published online: 19 June 2020

○) Springer Science+Business Media, LLC, part of Springer Nature 2020

\section{Correction to: \\ Bulletin of Environmental Contamination and Toxicology (2020) 104:820-827 \\ https://doi.org/10.1007/s00128-020-02878-x}

In the original publication of the article, there was an error in the abstract section. The incorrect sentence "the dominant microbes in the DEHP-contaminated soil were Sphingomonas and Bacillus, which belonged to the Acidobacteria and Proteobacteriav, respectively" should be revised to "the dominant microbes in the DEHP-contaminated soil were belonged to the Acidobacteria and Proteobacteria, respectively." This has been corrected with this erratum.
Publisher's Note Springer Nature remains neutral with regard to jurisdictional claims in published maps and institutional affiliations.

Cui Zhang and Peipei Song contributed equally to this work.

The original article can be found online at https://doi.org/10.1007/ s00128-020-02878-x.

Jun Wang

jwang@sdau.edu.cn

1 College of Resources and Environment, Key Laboratory of Agriculture Environment, Shandong Agricultural University, Tai' an 271018, People's Republic of China

2 Tai' an City Public Security Bureau in Shandong Province, Tai' an, People's Republic of China 